\title{
The establishment of rat model in myocardial ischemia with psychological stress
}

\author{
Chen Wang ${ }^{1,2 \#}$, Xiao-Reng Wang ${ }^{1 \#}$, Dan-Dan Song ${ }^{1}$, Jian-Li Wang ${ }^{1}$, You Wang ${ }^{1}$, Tian-Qi Tao ${ }^{1}$, Mi Liu ${ }^{1}$, \\ Xiu-Hua Liu ${ }^{1}$, Xu-Dong $\mathrm{Wu}^{3}$ \\ ${ }^{1}$ Department of Pathophysiology, Chinese PLA General Hospital, Beijing 100853, China; ${ }^{2}$ Institute of Basic Theory of Traditional Chinese \\ Medicine, China Academy of Chinese Medical Sciences, Beijing 100853, China; ${ }^{3}$ Out-patient Department, Chinese PLA General Hospital, Beijing \\ 100853, China \\ Contributions: (I) Conception and design: XH Liu, C Wang, XR Wang; (II) Administrative support: XH Liu, XD Wu; (III) Provision of study \\ materials or patients: XH Liu, XR Wang; (IV) Collection and assembly of data: C Wang, TQ Tao, DD Song, M Liu; (V) Data analysis and \\ interpretation: JL Wang, Y Wang; (VI) Manuscript writing: All authors; (VII) Final approval of manuscript: All authors. \\ \#These authors contributed equally to this work. \\ Correspondence to: Xiu-Hua Liu. Department of Pathophysiology, Chinese PLA General Hospital, Beijing 100853, China. Email: xiuhualiu98@163.com; \\ Xu-Dong Wu. Out-patient Department, Chinese PLA General Hospital, Beijing 100853, China. Email: wuxudong60 @163.com.cn.
}

Background: Psychological stress can provoke and aggravate myocardial ischemia, and this stress can even trigger acute coronary syndromes or sudden cardiac death. Therefore, for the first time, this study aimed to investigate the method for establishing a rat model of myocardial ischemia with psychological stress and its evaluation.

Methods: Forty male Wistar rats were randomly divided into the sham $(\mathrm{S}, \mathrm{n}=10)$, myocardial infarct (MI, n=10), psychological stress (MODEL, $\mathrm{n}=10)$, and myocardial infarct with psychological stress $(\mathrm{MI}+$ MODEL, $\mathrm{n}=10$ ) groups. The rat model of psychological stress was established by measuring the data from activity restriction for 6 hours and followed by tail clamp stimulation for 5 minutes every day for 14 days. The rat model of the myocardial infarct with psychological stress was established by occluding the left coronary anterior descending artery in the MODEL rats. The body weight of rats was measured daily, the behavior parameters were evaluated via open-field test and elevated plus-maze, tongue color and sublingual vein were observed, rats' acral blood flow perfusion was detected by PIM II (Perfusion Imager II), mesenteric microcirculation was measured by capillaroscopy, and hemodynamics was measured by a polygraph system. An automatic biochemical analyzer determined the content of serum cTnT (cardiac troponin T), Hcy (homocysteine), and activity of LDH (lactate dehydrogenase). Myocardial infarct size was measured with TTC (triphenyhetrazolium chloride) staining.

Results: We found that rats in the psychological stress (MODEL) group were characterized by coarse hair, dark mucosa of the lips and claw, low spirit, decreased body weight, and increased anxiety. Compared with rats in the sham group, rats in the MODEL + MI group showed decreased mesenteric blood flow, narrowed arteriole and venule diameter, reduced acral blood flow perfusion, and $L V \pm \mathrm{dp} / \mathrm{dt}_{\max }$ (the maximal rate of the increased and decrease of left ventricular pressure), as well as increased serum content of c'TnT, Hcy, and LDH activity. Compared with the MI group, rats in the MODEL + MI group showed deteriorated microcirculation dysfunction manifested as a dark tongue color of deep purple, prominently extended and varicose sublingual vein, and aggravated myocardial damage in the form of increased infarct size and LDH leakage.

Conclusions: In conclusion, the rat model of myocardial ischemia with psychological stress was successfully established, and manifested as aggravating behavioral disorder, mesenteric microcirculation and left ventricular dysfunction, and myocardial damage. 
Keywords: Myocardial ischemia; psychological stress; rat model

Submitted Sep 24, 2019. Accepted for publication Feb 07, 2020.

doi: $10.21037 /$ atm.2020.02.128

View this article at: http://dx.doi.org/10.21037/atm.2020.02.128

\section{Introduction}

Coronary heart disease (CHD) is still the disease with the highest mortality and morbidity worldwide. Despite the improvements in the surgical and pharmacological therapies for CHD, the mortality is still high. More than 6 decades of empirical research have shown that psychological risk factors contribute both to the risk of developing CHD and the worsening of the clinical course and prognosis in patients with CHD. Evidence shows that psychological stress-induced myocardial ischemia is associated with a 2 -fold increased risk of future cardiac events independent of physical stress-induced ischemia in patients with stable CHD $(1,2)$. These factors may act as barriers to treatment adherence and efforts to improve life-style in patients. It has been proven that psychological stress can cause endocrine imbalance; leading to subsequent glucose and lipid metabolism disorder; activation of the sympathetic nervous system with hemodynamic changes; induction of coronary and peripheral vasoconstriction, transient endothelial dysfunction, arterial stiffening, and inflammation reaction changes such as increased tumor necrosis factor- $\alpha$ (TNF- $\alpha$ ) and decreased secretory immunoglobin A (IgA) (3-11) which ultimately results in vessels stenosis, obstruction, and spasm.

Anxiety is the most common experience among the possible psychological stress factors that can hurt health outcomes, and an independent risk factor and predictor of CHD (12-14). Nonetheless, the negative role and mechanisms for developing anxiety in CHD patients are still unknown because of a lack of a reliable animal models for investigation. Therefore, a workable and efficient animal model of myocardial ischemia with psychological stress (anxiety) is needed. Peng et al. and Ren et al. used different chronic unpredictable emotional stimulation such as noise, diet, temperature, light, or tail clamp to establish a rat model of psychological stress $(15,16)$. Xu et al. proved that it was possible to develop a mice model of pure psychological stress by combined stimuli such as isolation stress, predator stress, and social failure stress (17). Jia et al. established a psychological stress mice model by applying the "observing electroshock experiment" (18). Zhang et al. compared different methods (maternal deprivation alone; chronic restriction alone and maternal deprivation combining restriction) that imitate psychological stress, and then combined them with diarrhea-causing factors to establish irritable bowel syndrome with psychological stress rat model (19). Li et al. established rat model of asthma with psychological stress through the sensitization of ovalbumin (OVA) mixture combing restriction (20). However, the application and evaluation of this model in CHD are still unknown. Therefore, our purpose is to establish a feasible and efficient rat model of myocardial ischemia with psychological stress and investigate its evaluation.

\section{Methods}

\section{Animal model}

All experimental animals were obtained from the Experimental Animal Center, Chinese PLA General Hospital (Beijing, China). Forty male Wistar rats, specific pathogen free (SPF), weighing $180 \pm 20 \mathrm{~g}$, were fed a normal diet provided by the same department and housed 10 per cage at $23{ }^{\circ} \mathrm{C}$ with luminosity cycles of 12 -hour light/ 12-hour dark and allowed free access to water and food. The protocol was approved by the Institutional Animal Care and Use Committee of the Chinese PLA General Hospital. All surgery was performed under sodium pentobarbital anesthesia. According to the recent references about the rat model of myocardial infarction, sodium pentobarbital is the most often used analgesic agent. Compared to chloral hydrate, isoflurane or urethane, sodium pentobarbital intraperitoneal injection has advantages of faster onset time, quick recovery effect, mild side effects, and lower lethality $(21,22)$. To make sure the analgesic effect was effective, we used a slightly larger dose of sodium pentobarbital, and all efforts were made to minimize suffering.

All rats were allowed free access to water and food for 14 days and randomly divided into 4 groups $(n=10)$ described below. (I) The psychological stress (MODEL) group involved the following: rats' activities were restricted using a binding device for 6 hours and followed by tail clamp stimulation using a binder clip for 5 minutes every day for 14 days. (II) The myocardial infarct (MI) 
group involved the following: rats were fixed on the operating table under anesthesia condition with $2 \%$ pentobarbital sodium peritoneally $(2.3 \mathrm{~mL} / \mathrm{kg})$; under artificial ventilation with a rodent ventilator, the thoracic cavity was opened at the third or fourth left intercostal space to expose the heart; the proximal part of the left coronary artery was surgically occluded through ligation with a suture (size 6.0); coronary occlusion was confirmed by electrocardiogram (ECG) obtained from a limb lead. (III) The myocardial infarct with psychological stress (MODEL + MI) group involved the following: rats were treated with restriction for 6 hours and followed by tail clamp stimulation for 5 minutes every day for 14 days followed by MI procedure as described in MI groups. (IV) The sham (S) group involved the following: the rats in sham group underwent the same surgical procedure excepting for the ligation of the left coronary artery. The rats were returned to their cages and killed 24 hours later.

\section{General information}

Rats' general condition of hair, mucosa of lips and claw, and activities were observed; body weight was measured daily.

\section{Behavior parameters}

\section{Open-field test}

An open-field test is considered a measurement related to anxiety (23). In order to evaluate this behavior, rats were placed in an open field arena $(80 \mathrm{~cm} \times 80 \mathrm{~cm} \times 50 \mathrm{~cm})$, divided into 25 squares $(20 \mathrm{~cm} \times 20 \mathrm{~cm})$ in a dimly lit and noise-free room as previous described (24). Briefly, rats were placed in an open field in the same location facing the same direction. After allowing for 10 seconds of adaptation, video capturing was undertaken for the next 5 minutes. The open field was cleaned with $75 \%$ alcohol gauze pads following each test. The time spent in the central squares was quantified, and horizontal movements through each square (crossing), the times of rearing, the times of face and body washing, and the number of defecations, were recorded by 2 individuals blinded to the experiment as an anxiety index.

\section{Elevated plus-maze}

The maze used was based primarily on the design of Costa et al. (25). The maze consisted of 2 open arms $(50 \mathrm{~cm} \times 10 \mathrm{~cm})$ and 2 enclosed arms $(50 \mathrm{~cm} \times 10 \mathrm{~cm} \times 40 \mathrm{~cm})$ without a roof. They intersected at the central square $(10 \mathrm{~cm} \times 10 \mathrm{~cm})$. It was elevated $50 \mathrm{~cm}$ from the floor. Rats were placed on the central square of the maze facing an open arm at the beginning of the test. Following 10 seconds of adaptation, video capturing was undertaken for the next 5 minutes, and behavioral parameters were obtained. The numbers and total time of entries to the open or enclosed arms were recorded and taken by 2 individuals blinded to the experiment as an anxiety index. An entry was considered as the moment the 4 paws of the rat were placed in the respective arm.

\section{Tongue inspection}

Twenty-four hours after MI, rats were anesthetized through intraperitoneal administration of $2.3 \mathrm{~mL} / \mathrm{kg} 2 \%$ sodium pentobarbital. The mouth was opened, and the tongue was pulled out gently. The tongue color and sublingual vein were recorded by the digital camera (D50, Nikon, Japan), and analyzed by 2 individuals blinded to the experiment.

\section{Acral blood flow perfusion}

After tongue inspection, acral blood flow perfusion was detected using Laser Doppler Perfusion Imager (LISCAPIMII, Sweden).

\section{Microscopy monitoring of mesenteric microcirculation}

Followed by acral blood flow perfusion measurement, rats' mesenteries were exposed immediately and put into an observation position in a small container that was filled with $37{ }^{\circ} \mathrm{C} 0.9 \% \mathrm{NaCl}$. Mesenteric microcirculation was monitored by the use of a biomicroscope (BH-2, Olympus, Japan) equipped with a video camera (BY-110, JVC, Japan) and a computer.

\section{Hemodynamic measurement}

Twenty-four hours after coronary occlusion, rats were anesthetized as described above. After an ECG was taken, a polyethylene tube (PE 50; Becton-Dickinson, U.S) was inserted into the left ventricular cavity via the right carotid artery. Left ventricular $\pm \mathrm{dp} / \mathrm{dt}_{\max }$ was measured using a polygraph system (AP601G; Nihon Koden, Japan).

\section{Content of serum cardiac troponin $T$ (cTnT), bomocysteine} $(\mathrm{Hcy})$, and activity of lactate dehydrogenase $(\mathrm{LDH})$

After the hemodynamic measurement, blood samples were collected from the right common carotid artery and the 


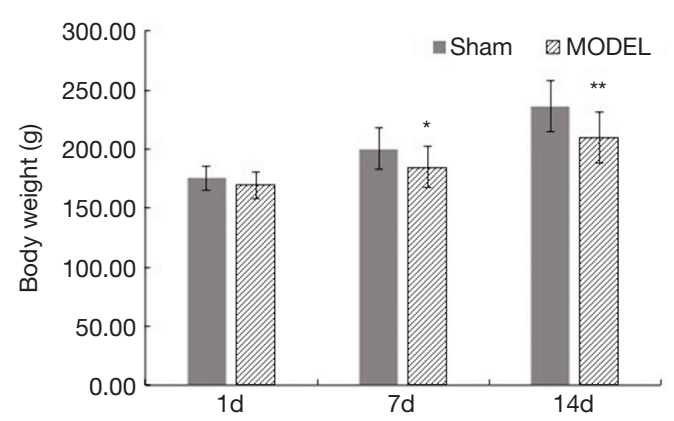

Figure 1 Effect of psychological stress on body weight ${ }^{*}, \mathrm{P}<0.05$ vs. sham group, ${ }^{* *}, \mathrm{P}<0.01$ vs. sham group, $\left.\mathrm{n}=10\right)$.

serum was separated by $1,000 \mathrm{rpm} / \mathrm{min}$ centrifugation for 10 minutes. The content of serum cTnT, Hcy, and activity of $\mathrm{H}$-type $\mathrm{LDH}$ was determined with an automatic biochemical analyzer (CObas8000, Roche, Germany).

\section{Infarct size}

Infarct size was measured using an established method (26). The coronary artery was again briefly occluded through ligation of the tie that remained at the site of the earlier occlusion. Immediately after the ligation, $5 \mathrm{~mL} 1 \%$ Evans blue solution was infused through the catheter into the beating left ventricular cavity to delineate the ischemic area at risk. After the administration of an excessive dose of sodium pentobarbital into the left ventricular cavity, the heart was excised and cross-sectioned from the apex to the atrioventricular groove into 5 specimens of $\sim 2 \mathrm{~mm}$ in thickness. These slices were incubated with a $4 \%$ TTC solution for 30 minutes at $37^{\circ} \mathrm{C}$ in a dark room. Then, the ischemic but viable, infarcted area and the non-ischemic area were fixed in $10 \%$ neutral-buffered formalin for 24 hours. The infarct zone (white) was an area of necrosis (AN, Evans blue and TTC-unstained); the ischemic but viable area (red) was the TTC-stained area, and the nonischemic area (blue) was Evans blue-stained. The area at risk (AAR) was the sum of white and red zones. These areas were measured with scanner and Image-Pro Plus (image analyzing software, Version 4.1, Media Cybernetics). Infarct size was defined as the ratio of AN/AAR.

\section{Statistical analysis}

The SPSS 13.0 version was adopted for statistical analysis. Results were expressed as means \pm standard deviation.
Multiple comparison analysis was performed with TuckeyKramer test. A P value of less than 0.05 was considered statistically significant.

\section{Results}

\section{General information}

Rats in the sham group were characterized by smooth hair, high spirit, and pink mucosa of the lips and claws. Rats in the MODEL group showed almost the same characterization as the rats in the sham group on the $1^{\text {st }}$ day, but on the $7^{\text {th }}$ and $14^{\text {th }}$ day, they were characterized by coarse hair, low spirit, and dark mucosa of the lips and claws. Also, compared with the bodyweight of rats in the sham group, that of the rats in the MODEL group on the $7^{\text {th }}$ day and $14^{\text {th }}$ day significantly decreased $(\mathrm{P}<0.05$ and $\mathrm{P}<0.01$, respectively) (Figure 1).

\section{Open-field test}

Compared with the sham rats, the MODEL rats showed a reduction in time spent in the central squares, horizontal movements through each square (crossing), times of rearing, and times of face and body washing, but increased defecation numbers. There was a significant difference in times of rearing and washing $(\mathrm{P}<0.05$ vs. sham group), indicating an increased anxiety in the open-field test (Figure 2).

\section{Elevated plus-maze}

Compared with the sham rats, the MODEL rats showed a reduction in time spent in the open arms and numbers of entries into the open arms, but there was no significant difference $(\mathrm{P}>0.05)$. Meanwhile, the time spent in the close arms significantly was increased among the MODEL rats $(\mathrm{P}<0.05$ vs. sham rats), suggesting that the anxiety increased in the elevated plus-maze (Figure 3).

\section{Tongue inspection}

The sham rats showed a normal pink tongue with light purple and a proper size of the sublingual vein; the MODEL and MI rats showed a slightly dark tongue with purple coloring and a moderately extended and varicose sublingual vein; and the MODEL + MI rats showed a dark tongue with deep purple coloring, and a visibly extended and varicose sublingual vein (Figure 4). 

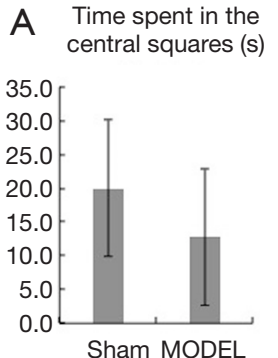

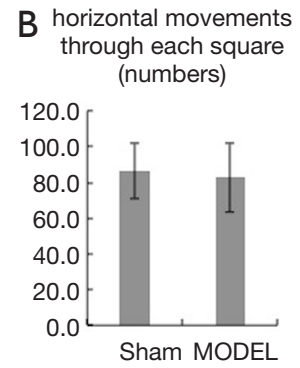

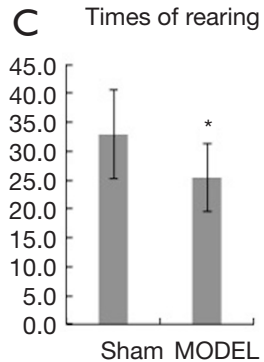

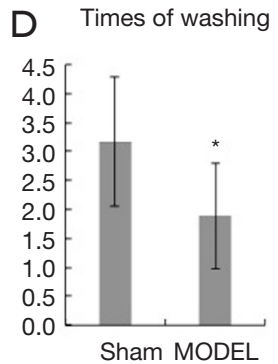

E Defecation (numbers)

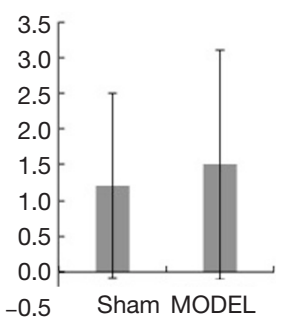

Figure 2 Effect of psychological stress on open-field test ( $\mathrm{n}=10)$. (A) Time spent in central squares; (B) horizon movements in each square; (C) times of rearing; (D) times of washing; (E) total numbers of defecation. Columns represent mean $\pm \mathrm{SEM}\left({ }^{*}, \mathrm{P}<0.05\right.$ vs. sham group).
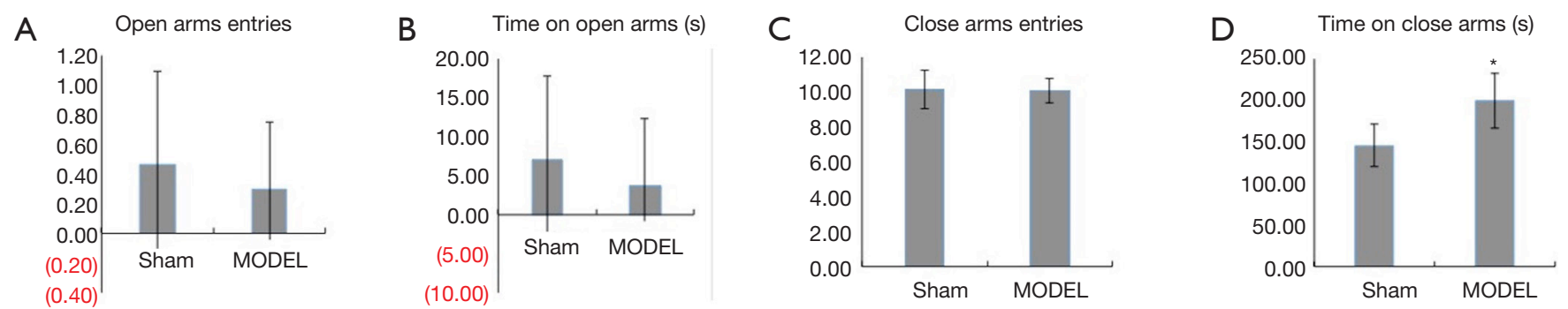

Figure 3 Effect of psychological stress on elevated plus-maze (n=10). (A) Entries into open arms; (B) time spent in open arms; (C) entries into closed arms; (D) time spent in closed arms. Columns represent mean \pm SEM $\left(^{*}, \mathrm{P}<0.05\right.$ vs. sham group).

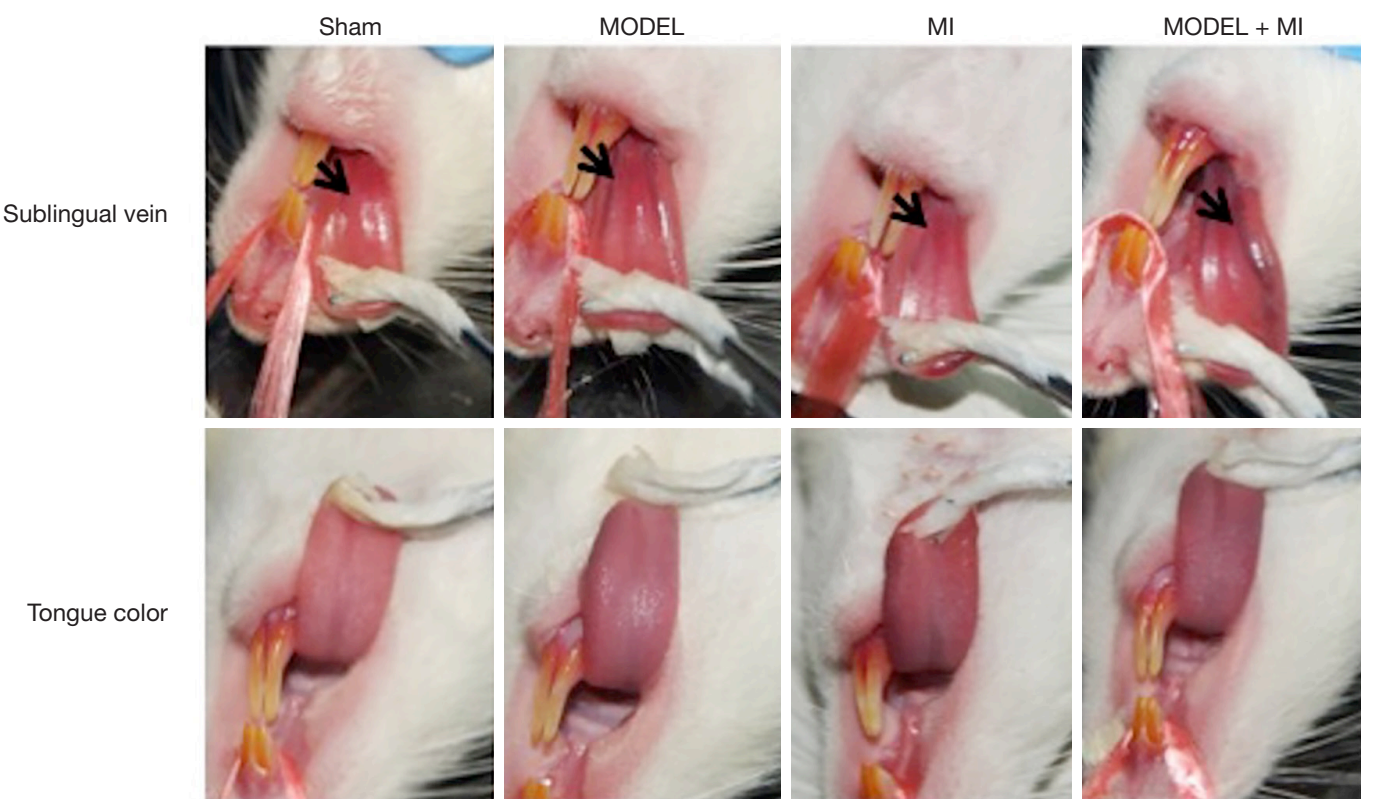

Figure 4 Effect of myocardium ischemia with psychological stress on tongue inspection. Arrow: sublingual vein. 


\section{Acral blood flow perfusion}

Compared with rats in the sham group, rats in MI, MODEL, and MODEL + MI groups all showed a significant decrease in acral blood flow perfusion $(\mathrm{P}<0.05)$ (Figure 5). Compared with rats in the MI group, acral blood flow perfusion of rats in the MODEL + MI group nonstatistically decreased $(\mathrm{P}>0.05)$.

\section{Microscopy monitoring of mesenteric microcirculation}

Compared with rats in sham group, rats in MI, MODEL and MODEL + MI groups all showed non-statistically decreased mesenteric blood flow speed $(\mathrm{P}>0.05)$, but a significantly narrowed arteriole diameter $(\mathrm{P}<0.05)$; the MODEL + MI rats also showed a narrowed venule diameter $(\mathrm{P}<0.05$ vs. the

\begin{tabular}{|c|c|c|c|c|}
\hline & Sham & MI & Model & Model + MI \\
\hline Mean & 2.154 & 1.714 & 1.609 & 1.555 \\
\hline SD & 0.410 & 0.337 & 0.270 & 0.314 \\
\hline
\end{tabular}

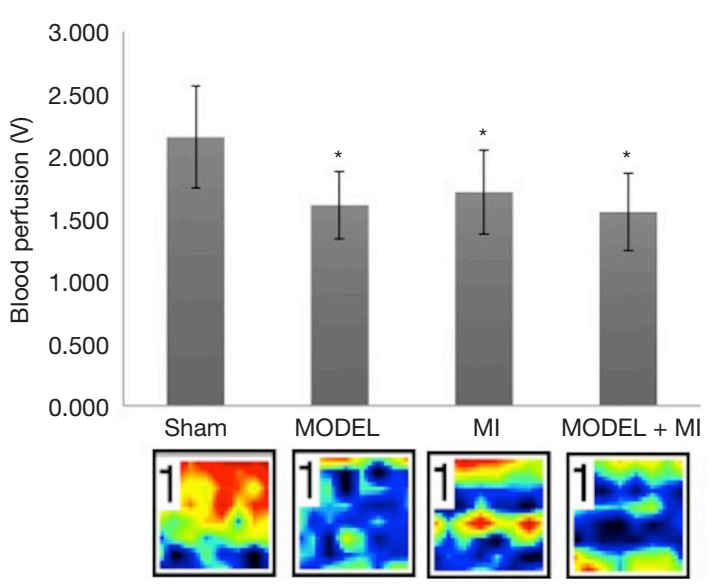

Figure 5 Effect of myocardium ischemia with psychological stress on acral blood perfusion ( ${ }^{*}, \mathrm{P}<0.05$ vs. sham group, $\left.\mathrm{n}=10\right)$. sham rats) (Figure 6). Compared with rats in the MI group, rats in the MODEL + MI group a showed non-statistically decreased arteriole and venule diameter $(\mathrm{P}>0.05)$.

\section{Hemodynamic measurement}

Compared with rats in the sham group, rats in both the MI and MODEL + MI group showed a significant decrease in $\mathrm{LV} \pm \mathrm{dp} / \mathrm{dt}_{\text {max }}(\mathrm{P}<0.05)$. The MODEL + MI rats showed a further decreased $+\mathrm{dp} / \mathrm{dt}_{\max }$ and $-\mathrm{dp} / \mathrm{dt}_{\max }$ of $13 \%$ and $9 \%$, respectively, compared with the MI rats, but this was not a significant difference $(\mathrm{P}>0.05)$ (Figure 7).

\section{Content of serum cTnT, Hcy, and activity of LDH}

Compared with rats in the sham group, the content of serum cTnT in the rats from MI and MODEL + MI groups non-statistically increased $(\mathrm{P}>0.05)$, while Hcy content and $\mathrm{LDH}$ activity in the rats from the MI and MODEL $+\mathrm{MI}$ groups showed a significant increase $(\mathrm{P}<0.05$ vs. sham group). Moreover, the MODEL + MI rats showed significantly increased LDH activity compared with MI rats $(\mathrm{P}<0.05)$ (Figure 8).

\section{Infarct size}

The infarct area was white, the area at risk was white and red, and the non-ischemia area was blue. The infarct size was the ratio of infarct area to the area at risk. The MI group was shown to have an infarct size $(80.95 \% \pm 2.45 \%)$. Compared with rats in the MI group, rats in the MODEL + MI group showed a further $12 \%$ increase in infarct size $(92.78 \% \pm 4.64 \%, \mathrm{P}<0.05)$ (Figure 9).

\section{Discussion}

Patients typically silently suffer from psychological stress-
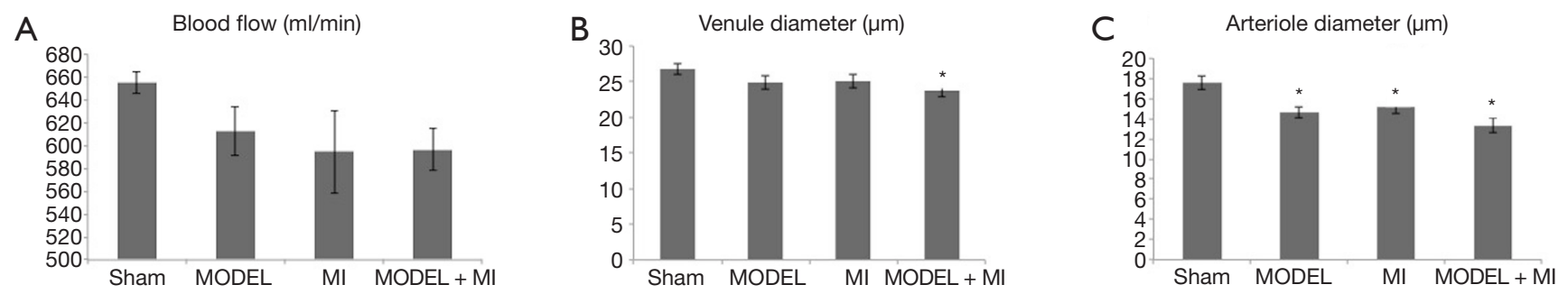

Figure 6 Effect of myocardium ischemia with psychological stress on mesenteric microcirculation. (A) Mesenteric blood flow; (B) mesenteric venule diameter; $(\mathrm{C})$ mesenteric arteriole diameter. Columns represent mean $\pm \mathrm{SEM}$ (*, $\mathrm{P}<0.05$ vs. sham group, $\mathrm{n}=10$ ). 

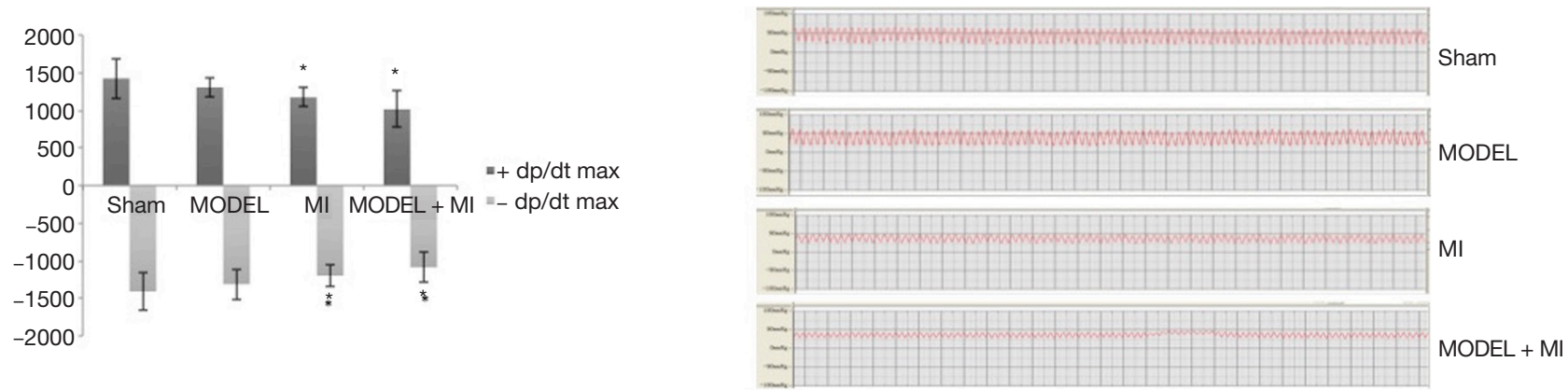

Figure 7 Effect of myocardium ischemia with psychological stress on $\mathrm{LV} \pm \mathrm{dp} / \mathrm{dt}_{\max }\left({ }^{*}, \mathrm{P}<0.05\right.$ vs. sham group, $\left.\mathrm{n}=10\right)$.
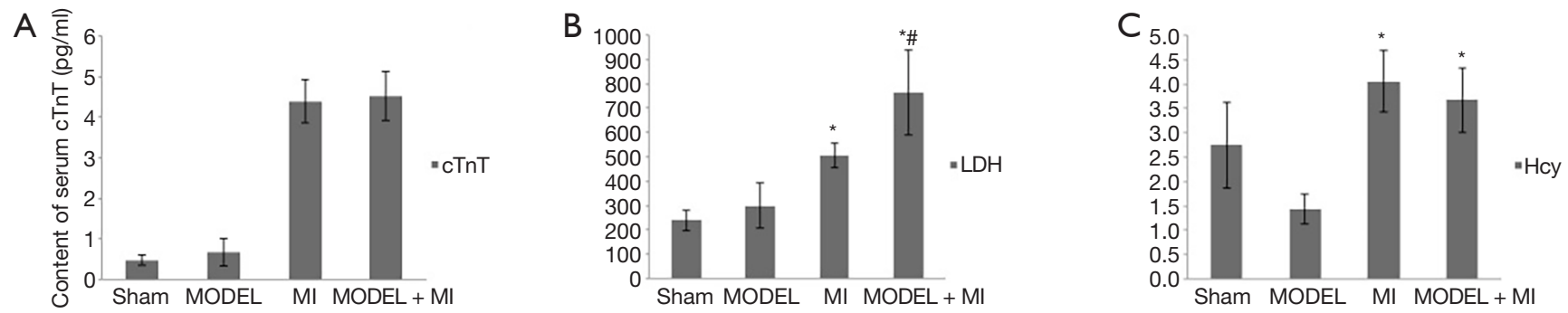

Figure 8 Effect of myocardium ischemia with psychological stress on serum cTnT, Hcy and LDH activity (*, P<0.05 vs. sham group;, $\mathrm{P}<0.05$ vs. MI group, $\mathrm{n}=10)$.

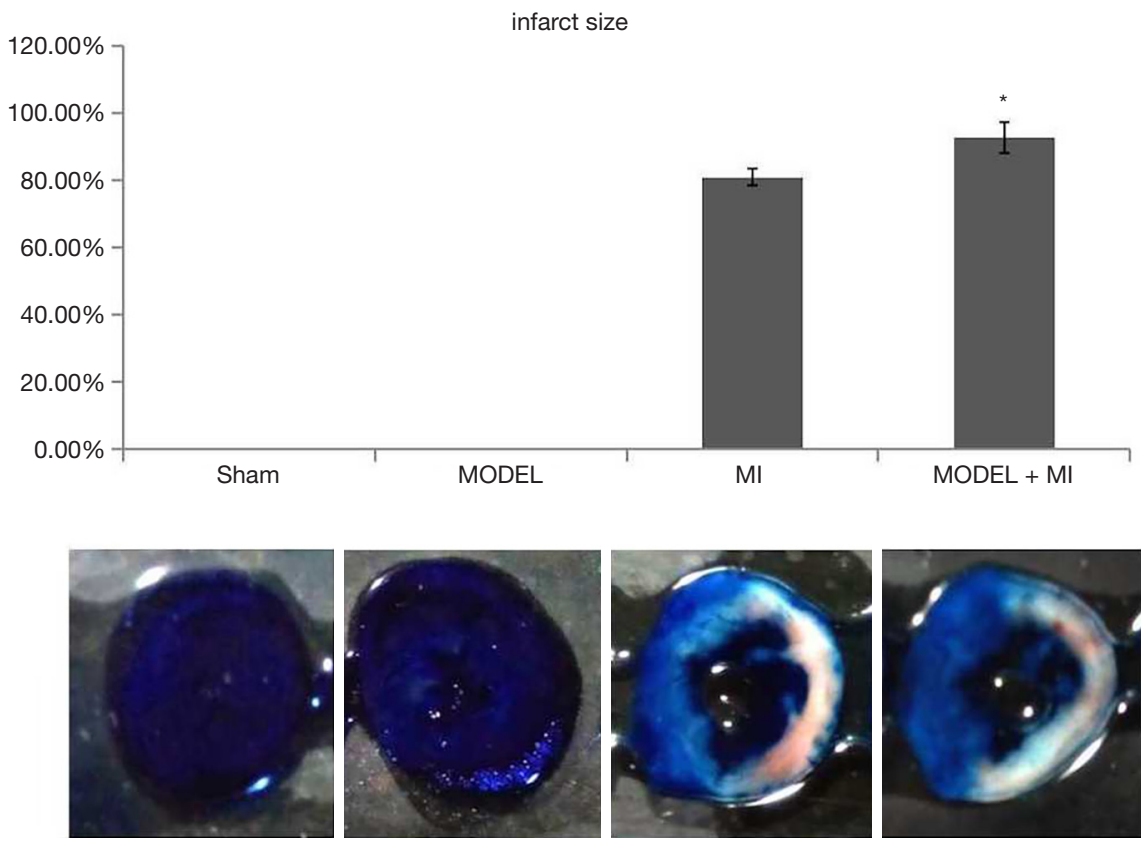

Figure 9 Effect of myocardium ischemia with psychological stress on myocardium infarct size (*, $\mathrm{P}<0.05$ vs. MI group, $\mathrm{n}=4)$. 
induced myocardial ischemia. Typically, it occurs at a lower workload than exercise-induced ischemia and is known to be associated with adverse cardiovascular outcomes, like cardiovascular death and MI. In the laboratory, psychological stress can aggravate ischemia that can be diagnosed as reversible left ventricular dysfunction, transient electrocardiographic abnormalities, or perfusion defects under nuclear imaging $(27,28)$.

Prolonged physical restriction results in psychological stress (29); therefore, we created the rat model using prolonged activity restriction. In addition, based on other studies of the psychological stress rat model, we created the time point (6 hours' activity restriction for 14 days) of our experiment. Psychological stress can be detected by changes in the rats' general condition and behavior parameters such as elevated plus-maze and open-field tests. In the present study, we established the model of myocardial ischemia with psychological stress by 6 hours' activity restriction and 5 minutes' tail clamp every day for 14 days, followed by myocardial ischemia by occluding the left anterior descending (LAD) for 24 hours in more readily irritated Wistar rats. In this model, we found, for the first time, that the rats which were subjected to psychological stress showed increased anxiety levels and behavioral disorders; meanwhile, compared with myocardial ischemia group alone, myocardial ischemia with psychological stress rats suffered more severe hemodynamic changes, microcirculation dysfunction, and increased infarct size, along with LDH leakage from myocardium, indicating aggravated myocardial injury in myocardial ischemia with psychological stress rats.

In CHD patients, tongue color and the sublingual vein are closely related to coronary artery stenosis and microcirculation dysfunction. The darker the tongue color (deep purple) and the more obvious the varicose sublingual vein are, the more severe the coronary artery stenosis and microcirculation dysfunction will be (30-33). Rats in either the psychological stress alone group or the myocardial infarct alone group showed a slightly darker tongue of light purple and a moderate varicose sublingual vein, while rats in the myocardial ischemia with psychological stress group showed a darker tongue color of deep purple and an obviously extended and varicose sublingual vein. Furthermore, rats in the myocardial ischemia with psychological stress group showed more severe microcirculation dysfunction such as decreased mesenteric blood flow, narrowed arteriole and venule diameter, and decreased acral blood flow perfusion. Taken together, these suggest deteriorated blood flow through blockage and circulation disorder.

Myocardial ischemia can directly cause cardiac dysfunction and myocardial damage, which can be reflected by left ventricular $\pm \mathrm{dp} / \mathrm{dt}_{\max }$ and chemical injury factor release. c $\operatorname{Tn} \mathrm{T}$ is a specialty biomarker to diagnose myocardial injury, and LDH activity is also applied to assess the cell injury; when cardiomyocytes are injured, the membrane becomes more permeable, which can lead to leakage of cTnT and LDH. Homocysteine is an independent risk factor in cardiovascular disease and closely related to the severity of Chinese medicinal syndromes of CHD (34). As we showed in our study, either myocardial infarct alone group or psychological stress with myocardial infarct group aggravated myocardial damage by decreasing $\mathrm{LV} \pm \mathrm{dp} / \mathrm{dt}_{\max }$ and increasing infarct size and serum chemical injury factors. Moreover, we found that compared with myocardial ischemia alone, psychological stress followed by myocardial ischemia further exacerbated myocardial damage, indicating that myocardial ischemia can be aggravated by psychological stress.

In conclusion, this study was the first to successfully establish a rat model in myocardial ischemia with psychological stress for the first time. However, there are still some limitations to this model, such as anxiety evaluation. We should extend the restriction time of our model in a future study, so that we can observe more severe pathological stress changes.

\section{Acknowledgments}

Funding: This project was supported by grants from National Basic Research Program of China (973 Program No. 2015CB554405 and No. 2015CB554402).

\section{Footnote}

Conflicts of Interest: The authors have no conflicts of interest to declare.

Ethical Statement: The authors are accountable for all aspects of the work in ensuring that questions related to the accuracy or integrity of any part of the work are appropriately investigated and resolved. The protocol was approved by the Institutional Animal Care and Use Committee of the Chinese PLA General Hospital (No. 2018-085). 
Open Access Statement: This is an Open Access article distributed in accordance with the Creative Commons Attribution-NonCommercial-NoDerivs 4.0 International License (CC BY-NC-ND 4.0), which permits the noncommercial replication and distribution of the article with the strict proviso that no changes or edits are made and the original work is properly cited (including links to both the formal publication through the relevant DOI and the license). See: https://creativecommons.org/licenses/by-nc-nd/4.0/.

\section{References}

1. Dhar AK, Barton DA. Depression and the Link with Cardiovascular Disease. Front Psychiatry 2016;7:33.

2. Wei J, Roobs C, Ramadan R, et al. Meta-analysis of mental stress-induced myocardial ischemia and subsequent cardiac events in patients with coronary artery disease. Am J Cardiol 2014;114:187-92.

3. Pimple P, Lima BB, Hammadah M, et al. Psychological Distress and Subsequent Cardiovascular Events in Individuals With Coronary Artery Disease. J Am Heart Assoc 2019;8:e011866.

4. von Känel R. Acute mental stress and hemostasis: when physiology becomes vascular harm. Thromb Res 2015;135:S52-5.

5. Ghaffari AS, Zhao W, Bibi S, et al. Statistical Analysis of Dispositional and Psychological Factors and their Association with Cardiovascular Diseases. Eur Online J Nat Soc Sci 2018;7:720-7.

6. Pedersen SS, von Kanel R, Tully PJ, et al. Psychosocial perspectives in cardiovascular disease. Eur J Prev Cardiol 2017;24:108-15.

7. Xue YT, Tan QW, Li P, et al. Investigating the role of acute mental stress on endothelial dysfunction: a systematic review and meta-analysis. Clin Res Cardiol 2015;104:310-9.

8. Cohen BE, Edmondson D, Kronish IM. State of the Art Review: Depression, Stress, Anxiety, and Cardiovascular Disease. Am J Hypertens 2015;28:1295-302.

9. Kamkar MZ, Sanagoo A, Zargarani F, et al. Metabolic syndrome in patients with severe mental illness in Gorgan. J Nat Sci Biol Med 2016;7:62-7.

10. Ma CY, JH Liu, JX Liu, et al. Relationship between two blood stasis syndromes and inflammatory factors in patients with acute coronary syndrome. Chin J Integr Med 2017;23:845-9.

11. Hammadah M, Sullivan S, Pearce B, et al. Inflammatory response to mental stress and mental stress induced myocardial ischemia. Brain Behav Immun 2018;68:90-7.

12. Du J, Zhang D, Yin Y, et al. The Personality and Psychological Stress Predict Major Adverse Cardiovascular Events in Patients with Coronary Heart Disease After Percutaneous Coronary Intervention for Five Years. Medicine (Baltimore) 2016;95:e3364.

13. Batelaan NM, Seldenrijk A, Bot M, et al. Anxiety and new onset of cardiovascular disease: critical review and metaanalysis. Br J Psychiatry 2016;208:223-31.

14. Stewart RAH, Colquhoun DM, Marschner SL, et al. Persistent psychological distress and mortality patients with stable coronary artery disease. Heart 2017;103:1860-6.

15. Peng WY, Mu LY, Qian PP. Establishment of psychological stress rat model, J Med Thero Prac 2002;15:625.

16. Ren JX, Lin CR, Wang M, et al. The establishment of psychological stress animal model with multi-elements combination. Pharmacology and Clinics of Chinese Materia Medica 2007;23:210-1.

17. Xu DB, Wang J, Yu YN, et al. Establishment of mice model of pure psychological stress by combined stimuli: an experimental study. Journal of Chongqing Medical University 2016;41:832-5.

18. Jia KR, Guo G, Liu KY, et al. Establishment of an animal model with pure psychological stress and its effects on behaviour and endocrine-immune function. Immunol J 2009;25:329-32.

19. Zhang BH, Wang W, Wang FY, et al. Comparative study of establishment of a rat model of IBS-D based on different psychological stress. World Chinese Medicine 2017;12:2128-33.

20. Li CA, Hu XL, Cui HS, et al. Establishment and evaluation of psychological stress asthmatic rat model. Journal of Beijing University 2016;39:1033-40.

21. Kezeli T, Rukhadze T, Gongadze N, et al. Effect of calcitonin gene-related peptide antagonist on the cardiovascular events, mortality, and prostaglandin E2 production by nitrate-induced tolerant rats with acute myocardial infarction. EPMA J 2016;7:6.

22. Yang TT, Xiao Y, Xi SF, et al. Improvement of the methods of establishing and evaluating rat models of myocardial infarction. Chinese Journal of Comparative Medicine 2014;24:46-51.

23. Kuniishi H, Ichisaka S, Yamamoto M, et al. Early deprivation increases high-leaning behavior, a novel anxiety-like behavior, in the open field test in rats. Neurosci Res 2017;123:27-35.

24. Ginty AT, Kraynak TE, Fisher JP, et al. Cardiovascular 
and autonomic reactivity to psychological stress:

Neurophysiological substrates and links to cardiovascular disease. Auton Neurosci 2017;207:2-9.

25. Costa AA, Morato S, Roque AC, et al. A computational model for exploratory activity of rats with different anxiety levels in elevated plus-maze. J Neurosci Methods 2014;236:44-50.

26. Lichtenauer M, Schreiber C, Jung C, et al. Myocardial infarct size measurement using geometric angle calculation. Eur J Clin Invest 2014;44:160-7.

27. Vaccarino AL, Evans KR, Kalali AH, et al. The Depression Inventory Development Work group: A Collaborative, Empirically Driven Initiative to Develop a New Assessment Tool for Major Depressive Disorder. Innov Clin Neurosci 2016;13:20-31.

28. Arri SS, Ryan M, Redwood SR, et al. Mental stressinduced myocardial ischaemia. Heart 2016;102:472-80.

29. Wirtz PH, von Känel R. Psychological Stress, Inflammation, and Coronary Heart Disease. Curr Cardiol Rep 2017;19:111.

Cite this article as: Wang C, Wang XR, Song DD, Wang JL, Wang Y, Tao TQ, Liu M, Liu XH, Wu XD. The establishment of rat model in myocardial ischemia with psychological stress. Ann Transl Med 2020;8(6):322. doi: 10.21037/atm.2020.02.128
30. Liu Y, An DQ. Research on the relationship between sublingual vein and vascular diseases. Hunan Journal of Traditional Chinese Medicine 2014;30:135-6.

31. Hu LL, Li YY, Zhao QQ, et al. Research and expectation of relationship of tongue inspection and degree of coronary artery disease. Cardiovascular Disease Electronic Journal of integrated traditional Chinese and Western Medicine 2019;7:20-1

32. Qi L, Zhang JH. The Correlation of Sublingual Collaterals and Coronary CTA. Acta Chinese Medicine 2016;31:1586-8.

33. Ma H, Guo L, Huang D, et al. The Role of the Myocardial Microvasculature in Mental Stress-Induced Myocardial Ischemia. Clin Cardiol 2016;39:234-9.

34. Karadeniz M, Sarak T, Duran M, et al. Hyperhomocysteinemia Predicts the Severity of Coronary Artery Disease as Determined by the SYNTAX Score in Patients with Acute Coronary Syndrome. Acta Cardiol Sin 2018;34:458-63. 